\title{
Primer reporte de necrosis avascular de cabeza femoral en una cabra (Capra aegagrus hircus) en el Perú
}

\author{
Eben Salinas $C^{1 *} \otimes(\mathbb{D})$ M.Sc; Irma Celi $M^{1} \bowtie(D)$ Ph.D.
}

${ }^{1}$ Universidad Científica del Sur, Lima, Perú. Carrera de Medicina Veterinaria y Zootecnia. Panamericana Sur Km 19, Lima 42. Perú.

*Correspondencia: esalinasc@ucientifica.edu.pe

Recibido: Agosto 2018; Aceptado: Mayo 2019; Publicado: Septiembre 2019.

\section{RESUMEN}

Se reporta el caso de una cabra macho de raza alpina de 7 meses de edad, que presentaba claudicación del miembro posterior derecho sin causa definida. Se realizaron los exámenes clínicos y radiográficos, sugiriendo luxación traumática o necrosis avascular de cabeza femoral con consecuente enfermedad degenerativa articular. Se realizó tratamiento quirúrgico mediante artroplastia por excisión de cabeza y cuello femoral. El estudio histopatológico mediante la coloración de hematoxilina y eosina describió: hueso necrótico, lagunas conteniendo osteocitos necróticos, además de médula ósea necrótica. Esta información comprobó el diagnóstico de necrosis avascular de la cabeza y cuello femoral. En el post quirúrgico, la recuperación clínica del paciente se consideró buena, mitigando el dolor y mejorando la función del miembro afectado. El presente caso se trata del primer reporte de esta enfermedad en caprinos en el Perú.

Palabras clave: Aséptica, caprino, exéresis, osteoartritis, osteonecrosis (Fuente: DeCS).

\begin{abstract}
It is reported the case of a 7-month-old male goat of alpine race, who presented claudication of the right hind limb without definite cause. Clinical and radiographic examinations were performed, suggesting traumatic dislocation or avascular necrosis of the femoral head with consequent degenerative joint disease. It was performed a surgical treatment by an arthroplasty by excision of the head and neck of femur. The histopathological study by staining hematoxylin and eosin described: necrotic bone, lacunae containing necrotic osteocytes, as well as necrotic bone marrow. This information proved the diagnosis of avascular necrosis of the femoral head and neck. In the post-surgical period, the clinical recovery of the patient was considered good, the pain was mitigated and the function of the affected limb was improved. The present case is the first report of this disease in goats in Peru.
\end{abstract}

Keywords: Aseptic, goat, exeresis, osteoarthritis, osteonecrosis (Source: DeCS).

Como citar (Vancouver).

Salinas CE, Celi MI. Primer reporte de necrosis avascular de cabeza femoral en una cabra (Capra aegagrus hircus) en el Perú. Rev MVZ Cordoba. 2019; 24(3):7372-7377. DOI: https://doi.org/10.21897/rmvz.1431

(c) (1) (1) El (los) autor (es), Revista MVZ Córdoba 2019. Este artículo se distribuye bajo los términos de la licencia internacional Creative Commons

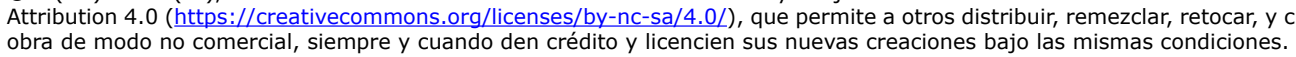




\section{INTRODUCCIÓN}

La necrosis avascular de la cabeza y cuello femoral (NACF), también es conocida como enfermedad de Legg-Calvé-Perthes o necrosis aséptica de la cabeza femoral. Esta es una enfermedad relacionada con el desarrollo y está descrita en los seres humanos, en animales de compañía y en diversas especies utilizadas como modelos animales $(1,2)$.

En los perros, la NACF tiene una presentación generalmente unilateral con una frecuencia de hasta el $80 \%$ de los casos (1). Las causas no son aún muy conocidas, sin embargo, la fisiopatología está descrita con un déficit en el suministro de sangre que causa isquemia y necrosis de la cabeza femoral. El déficit de suministro de sangre en esta zona puede deberse a diversos factores, en el caso de lesiones traumáticas en el cuello del fémur, el suministro de sangre y drenaje de la cabeza se verá comprometido por la ruptura de vasos en el sitio de la fractura (3). De manera experimental la NACF se ha reproducido usando corticoides (4) y producto de lesiones por agentes físicos como: temperatura, $\mathrm{pH}$, soluciones alcohólicas y la ligadura de las arterias circunflejas laterales y mediales (5). En el desarrollo de la NACF las fuerzas repetitivas que se ejercen sobre el hueso necrótico, a nivel de la epífisis de la cabeza femoral, causarán hendiduras y fisuras en el cartílago articular. Esto produce el colapso de las trabéculas subcondrales, seguido del aplanamiento progresivo de la cabeza femoral. Posteriormente, se da la reparación a través del tejido fibrovascular comenzando en la periferia de la cabeza femoral. Finalmente, la incongruencia articular conduce a la osteoartritis de la articulación coxofemoral (6)

Los signos clínicos que comúnmente se muestran son: claudicación unilateral o bilateral de los miembros posteriores, que progresa con la incapacidad para soportar el peso sobre la extremidad afectada o ambas extremidades. La palpación de la cadera puede generar dolor y dificultad de realizar los movimientos de abducción, flexión y extensión (3). Además, la extremidad afectada puede acortarse, el trocánter mayor se vuelve prominente y hay atrofia de los músculos glúteos y cuádriceps. (6)

El diagnóstico se puede complementar tomando radiografías de la cadera en incidencias ventrodorsal y mediolateral. El primer indicador radiográfico es el ensanchamiento de la cadera. La cabeza femoral afectada tiene una apariencia de vesícula delimitada a medida que el interior del hueso comienza a estar necrótico. La epífisis se muestra aplanada por la erosión subcondral y si hay destrucción trabecular suficiente, la cortical empieza a romperse y colapsarse (7). En este momento la cabeza femoral puede aparecer fragmentada. Finalmente, en el proceso de cicatrización, la cabeza femoral no llega a su forma original; causando que el acetábulo intente acomodarse y se vuelva artrítico (1).

El tratamiento generalmente está dirigido al control del dolor y a evitar la pérdida de función de la extremidad afectada. De manera conservadora, se plantea el reposo completo y tratamiento con antiinflamatorios no esteroideos. También está reportado el reemplazo completo de la cabeza y cuello femoral (8). Sin embargo, la artroplastia por excisión de la cabeza y cuello femoral, es la técnica más utilizada como rescate del miembro afectado y destinada a aliviar el dolor. No obstante, se ha reportado pseudoartrosis y otras complicaciones lo cual puede conducir a resultados impredecibles (9).

\section{EVALUACIÓN DEL PACIENTE}

Anamnesis. Se presentó a las instalaciones de la Universidad Científica del Sur, un paciente de especie caprina de sexo macho de 7 meses de edad y $18 \mathrm{~kg}$ de peso, con historial de aparente lesión por trauma en la cadera. El animal presentaba claudicación con apoyo del miembro posterior derecho. El animal era natural de Lima Metropolitana y convivía en un potrero con otros caprinos de igual edad y tamaño.

Hallazgos al examen clínico. En el examen clínico se evaluó al paciente en estación, al caminar y al trote. El paciente presentó claudicación de grado 2 del miembro posterior derecho (en una escala de 0 a 5: cojera moderada y claramente visible en el trote). A la inspección de la pelvis se observó hipotrofia muscular de la cadera, así como del miembro posterior derecho. Además presentó una notoria prominencia de la región lateral sobre el trocánter mayor del fémur (Figura 1).

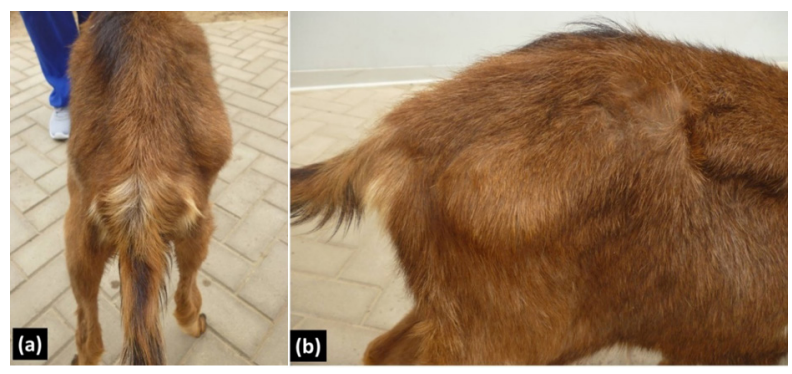

Figura 1. Vista posterior (a) y lateral derecha (b) en una cabra alpina macho de 7 meses de edad con claudicación del miembro posterior derecho: Se identifica una prominencia en la región lateral sobre el trocánter mayor del fémur 
La evaluación física se realizó con sedación, identificándose dificultad para realizar la extensión y flexión de la articulación de la cadera derecha y detectándose sonidos crepitantes en la articulación coxofemoral. Se realizó la maniobra de Ortolani resultando positiva. Con esta información se planteó el diagnostico presuntivo de luxación traumática o necrosis avascular de cabeza y cuello femoral con consecuente enfermedad degenerativa articular.

Ayudas diagnósticas. Con el objetivo de complementar el diagnóstico clínico, se realizaron radiografías de pelvis en dos incidencias (medio lateral y ventro dorsal). Se observó en la articulación coxofemoral derecha, aumento del espacio articular, forma irregular de la cabeza y cuello femoral y osteofitosis peri articulares (Figura 2).
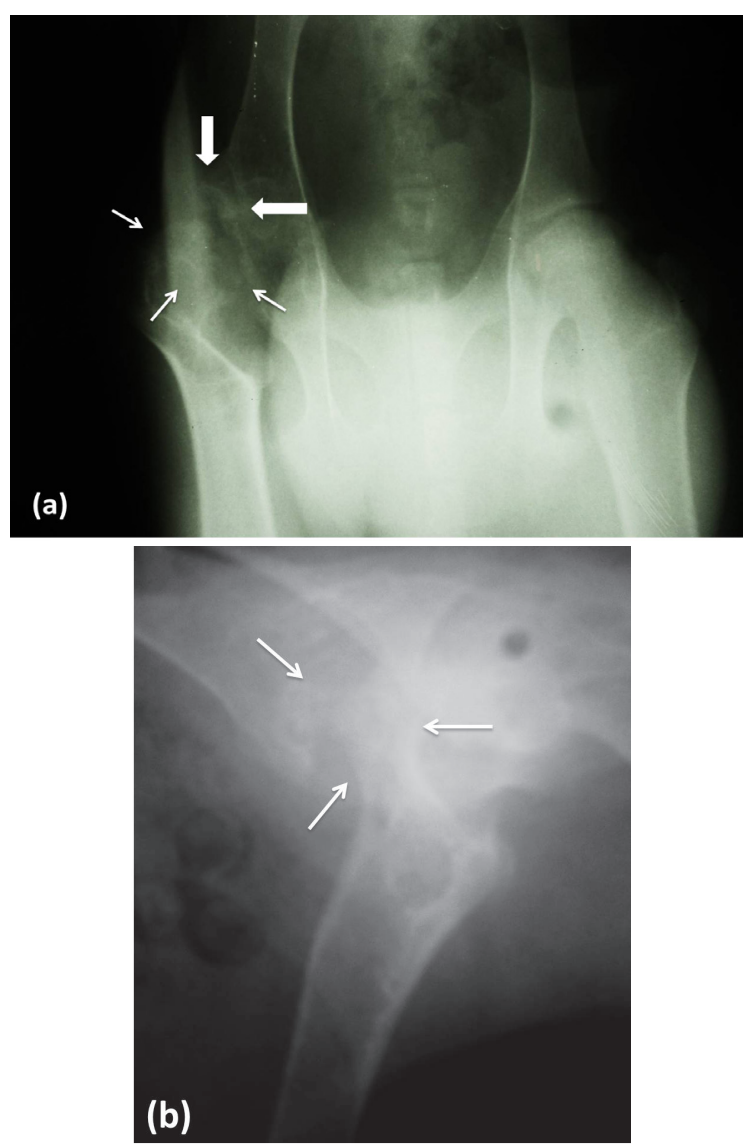

Figura 2. Radiografía de cadera en incidencia ventro dorsal (a) y medio lateral (b) de un caprino alpino macho de 7 meses de edad. Articulación coxofemoral derecha: irregularidad y aspecto poroso de la cabeza femoral con áreas de densidad disminuida en la epífisis, compatible con lisis ósea focal (flechas delgadas). La zona cráneo - lateral del acetábulo presenta osteofitos (flechas gruesas). Aplanamiento e irregularidad de la superficie articular de la cabeza del fémur que causa subluxación de la articulación.
Enfoque de tratamiento. Sobre la base de los hallazgos del examen clínico y el estudio por imágenes se decidió realizar una artroplastía por excisión de la cabeza y cuello femoral derecho. Para la medicación pre-anestésica se aplicó medetomidina (1 $\mathrm{mg} / \mathrm{kg})$, ketamina $(2 \mathrm{mg} / \mathrm{kg})$ y butorfanol $(0.1 \mathrm{mg} /$ $\mathrm{kg}$ ) por vía muscular. Se canuló la vena cefálica y se realizó la inducción con Propofol $(2 \mathrm{mg} / \mathrm{kg}$ ) y Fentanilo $(0.001 \mathrm{mg} / \mathrm{kg})$. Una vez obtenida la inconciencia, se intubó al paciente y se mantuvo el plano anestésico con isofluorano en oxígeno al $100 \%$ utilizando un circuito de no reinhalación. Se realizó ventilación asistida durante la cirugía.

Se posicionó al paciente en decúbito lateral izquierdo, se realizó una incisión vertical cráneo lateral al trocánter mayor derecho. Luego de la disección del tejido subcutáneo, se realizó la retracción del músculo bíceps femoral caudalmente y el músculo tensor de la fascia lata cranealmente. Se realizó una incisión en la inserción perióstica del músculo vasto lateral y se reflejó ventralmente. Se identificó y se incidió la cápsula articular, la cual estaba muy engrosada, para luego realizar la sección del ligamento redondo. A la inspección de la cabeza femoral, esta presentó una superficie irregular y áspera al tacto; el cartílago articular estaba engrosado y mostraba ulceraciones y numerosas grietas subcondrales (Figura 3 ).

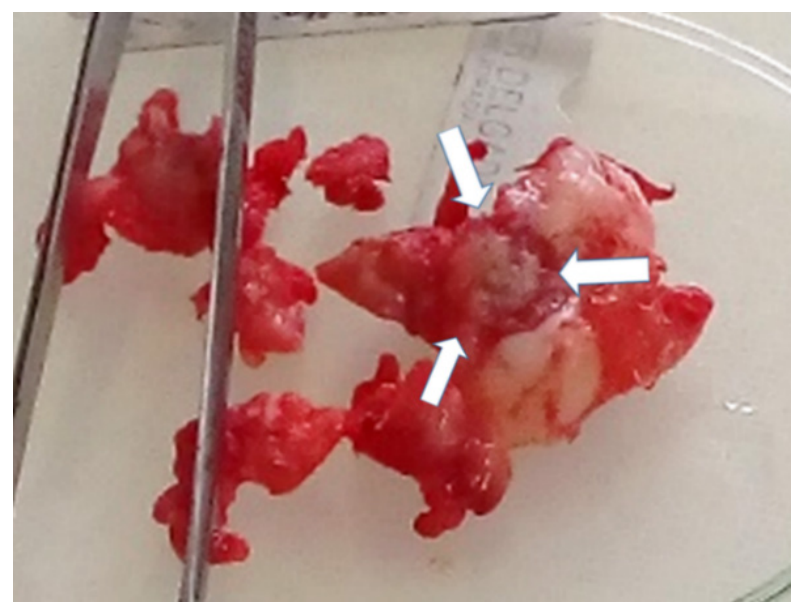

Figura 3. Pieza quirúrgica luego de la exéresis de cabeza y cuello femoral. Se nota el tejido multifragmentado con tejido irregular fibroso, en la cabeza femoral se evidencia una región de cartílago articular colapsado y necrótico (flechas blancas), así como grietas subcondrales.

Para realizar la ostectomía, un ayudante giró la extremidad hacia fuera hasta que la patela estuvo dirigida hacia arriba; esta maniobra produjo la luxación de la articulación. La exéresis se realizó con un osteótomo y un impactador. La línea de ostectomía correspondió a una línea recta entre la unión del cuello femoral y el tercer trocánter (Figura 4). 


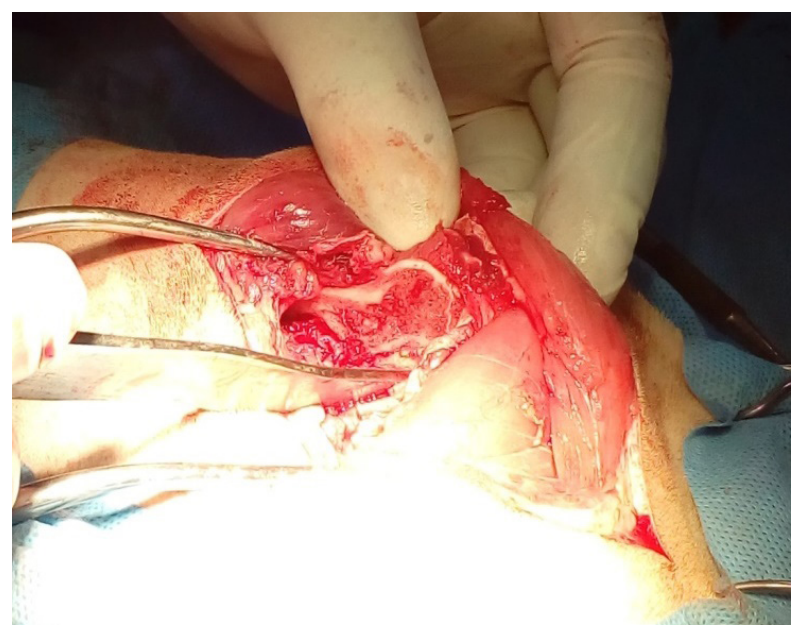

Figura 4. Se verificó la línea de incisión luego de la exéresis de cabeza y cuello femoral derecha.

Se realizó el cierre de los tejidos blandos con material de sutura absorbible calibre 1-0 y la sutura de piel con material no absorbible 1-0. El manejo del dolor se realizó con ketoprofeno a dosis de $1 \mathrm{mg} / \mathrm{kg}$ vía intramuscular cada 24 horas y antibióticoterapia con cefalotina a dosis de 30 $\mathrm{mg} / \mathrm{kg}$ vía intramuscular cada 12 horas. El paciente se trasladó a recuperación en jaula, iniciando su rehabilitación a las 24 horas con caminatas controladas, promoviendo el uso temprano de la extremidad. La recuperación fue considerada buena, logrando la sustentación completa del miembro operado y sin signos de dolor a los 30 días poscirugía.

Diagnóstico histopatológico. La pieza quirúrgica de cabeza y cuello femoral fue fijada en formol al $10 \%$ y remitida para su estudio histopatológico con la coloración hematoxilina y eosina en el Laboratorio de histopatología de la Universidad Científica del sur. Se observó una amplia variedad de necrosis del hueso trabecular con cambios inflamatorios limitados. El diagnostico correspondió a necrosis avascular de cabeza y cuello femoral (Figuras 5 y 6 ).

\section{DISCUSIÓN}

Los caprinos y otros rumiantes menores destacan por su anatomía y vasculatura similar a los seres humanos, por lo que han sido utilizados como modelos experimentales para el conocimiento y manejo de la $\operatorname{NACF}(2,10,11)$. Además, se ha utilizado como referencia al fémur de estos animales para conocer aspectos sobre la evolución y regeneración de la NACF (12). Sin embargo, no se encontraron reportes en la literatura consultada de casos experimentales y naturales de la NACF en caprinos en el Perú; por lo que consideramos el presente caso como un primer reporte.

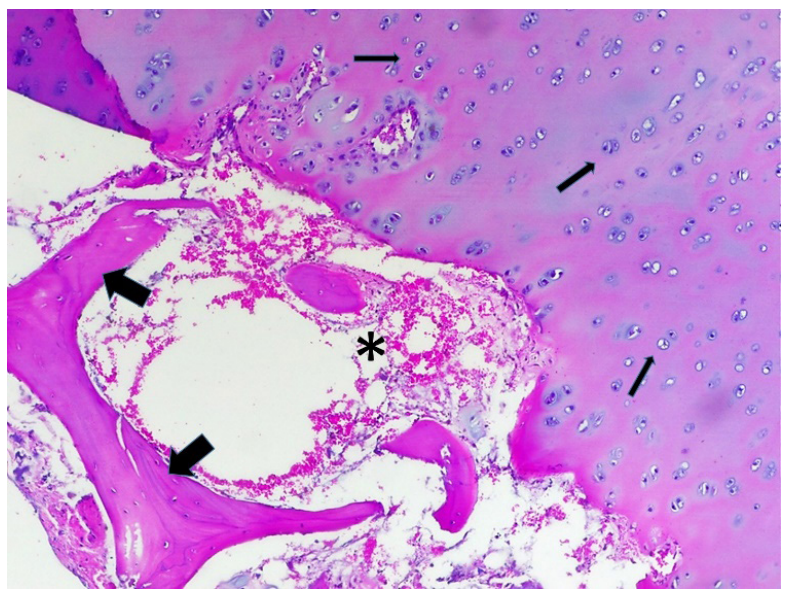

Figura 5. Sección de hueso necrótico, el cartílago articular está engrosado y se observan disposiciones anormales de los condrocitos (flechas delgadas). Área de necrosis ósea subcondral, se observa lagunas vacías del hueso trabecular conteniendo osteocitos necróticos (flechas gruesas). Los tejidos hematopoyéticos muestran depresión y se pueden apreciar tejidos adiposos atróficos (asterisco) (HE, 20x).

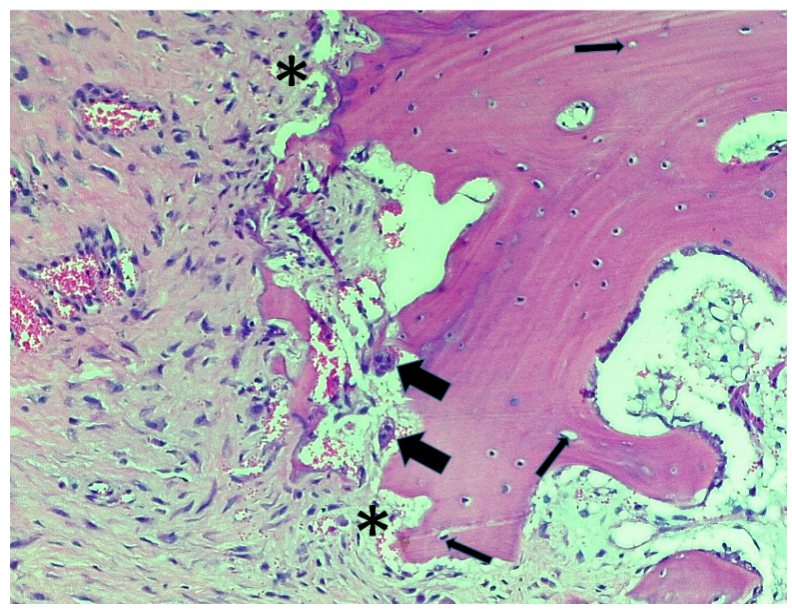

Figura 6. La médula ósea ha sido reemplazada por médula fibrótica en una matriz eosinofílica, este tejido en conjunto está parcialmente conectada al hueso necrótico (asteriscos). Algunas células gigantes multinucleados se pueden ver en la periferia de la matriz (flechas grandes). Se observan algunas lagunas vacías en el hueso trabecular conteniendo osteocitos necróticos (flechas delgadas). (HE, 40x).

La NACF no se encuentra bien descrita en pequeños rumiantes, por lo que se infiere que podría tener un componente hereditario y afectar más a animales jóvenes, como se reconoce en otras especies (1). Una probable explicación de los escasos reportes de la enfermedad puede ser consecuencia de las características propias de la cría de los caprinos (en rebaños o cría extensiva). Es común que los 
animales afectados con cojeras crónicas y que sean poco productivos, sean descartados o puedan finalmente padecer caquexia y muerte posterior sin causa definida. En ese sentido, también es posible que la NACF esté subdiagnosticada en cabras y sea confundida con enfermedades como la artritis encefalitis caprina y otras condiciones que conlleven a enfermedad degenerativa de las articulaciones (13).

En el presente caso, la herramienta utilizada para aproximar el diagnóstico de NACF fue la radiografía simple, la cual demostró densidad ósea irregular y cambios degenerativos propios de la osteonecrosis de la cabeza y cuello de fémur. Radiológicamente se observaron signos de osteonecrosis, con disminución de la radiodensidad de la cabeza femoral y contornos anormales de la misma (1). Estos hallazgos concuerdan con los hallazgos disponibles en estudios experimentales en cabras y ovejas (12).-

Los hallazgos histológicos correspondieron también a los hallazgos esperados en un hueso necrótico afectado por NACF $(11,14)$. Estos cambios fueron reportados como lagunas de osteocitos vacías o con osteocitos necróticos. Este hueso necrótico se vio rodeado de medula ósea fibrótica, la cual se vio desorganizada y con presencia de abundantes fibras colágenas. Se identificó además en la medula una matriz eosinofílicas y con algunas células gigantes multinucleadas adyacentes a las trabéculas óseas.

En el presente reporte, la cirugía se realizó con el objetivo de controlar el dolor en la articulación coxofemoral afectada, al limitar el contacto óseo inestable entre cabeza femoral y el acetábulo. Esta intervención generó la formación de una articulación falsa fibrosa, mejorando así la comodidad y la función del miembro afectado $(15,16)$. Se considera importante resaltar la atención postoperatoria en este caso, en el cual se promovió el uso de la extremidad lo más pronto después de la cirugía. Con este fin se realizaron caminatas diarias usando un arnés acondicionado al paciente, así como la aplicación de analgésicos para garantizar la comodidad y movilidad adecuada. El objetivo de esta medida se sustentó en evitar que el tejido fibroso que se formará durante el período postoperatorio, sea restrictivo y pueda limitar el rango de movimiento de la articulación coxofemoral intervenida (9).

Por otra parte, se podría considerar que la edad y el peso fueron factores adicionales que contribuyeron con la recuperación del paciente, ya que en perros y gatos, por ejemplo, se ha indicado que a mayor edad y mayor peso, se disminuye la capacidad de compensar las desventajas mecánicas en la articulación coxofemoral después de realizada la cirugía (8).

No se encontraron estudios controlados que hubieran evaluado los resultados quirúrgicos de la exéresis completa de la cabeza y cuello femoral en caprinos ni en otros rumiantes menores. Por lo tanto, se considera el presente caso como un primer reporte en Perú de NACF en caprinos.

\section{Conflicto de intereses}

Los autores declaran que no tienen ningún conflicto de intereses.

\section{Agradecimientos}

A las M.V.Z. Astrid Morillo, Brenda Benavides y Claudia Maguiña de la unidad de cirugía y ortopedia de la UCSUR; las cuales contribuyeron en el desarrollo del presente reporte.

\section{REFERENCIAS}

1. Jankovits DA, Liska WD, Kalis RH. Treatment of avascular necrosis of the femoral head in small dogs with micro total hip replacement. Vet Surg. 2012; 41(1):143-147. https://doi. org/10.1111/j.1532-950x.2011.00925.x
2. Boss JH, Misselevich I. Osteonecrosis of the Femoral Head of Laboratory Animals: The Lessons Learned from a Comparative Study of Osteonecrosis in Man and Experimental Animals. Vet Pathol. 2003; 40:345-354. https://doi.org/10.1354/vp.40-4-345 
3. Cardoso $C B$, Rahal SC, Mamprim MJ, Salvador $\mathrm{H}$, Melchert A, Figueroa J, et al. Avascular Necrosis of the Femoral Head in Dogs - Retrospective Study. Acta Scientiae Veterinariae. 2018; 46:1537. https://doi. org/10.22456/1679-9216.81845

4. Xie $X H$, Wang $X L$, Yang $H L$, Zhao DW, Qin L. Steroid-associated osteonecrosis: Epidemiology, pathophysiology, animal model, prevention, and potential treatments (an overview). J Orthop Translat. 2015; 3(2):58-70. https://doi.org/10.1016/j. jot.2014.12.002

5. Yang S, Wu X, Mei R, Yang C, Li J, Xu W, Ye S. Biomaterial-loaded allograft threaded cage for the treatment of femoral head osteonecrosis in a goat model. Biotechnol Bioeng. 2008; 100(3):560-56. https://doi.org/10.1002/ bit. 21792

6. DeCamp CE, Johnston SA, Déjardin LM, Schaefer SL. The hip joint. In: Piermattei D.L. (Ed). Brinker, Piermattei, and Flo's handbook of small animal orthopedics and fracture repair. 5th edn. St. Louis: Elsevier; 2016. https://doi.org/10.1016/b978-1-4377-23649.00025-2

7. Scherzer $C$, Windhagen $H$, Nellesen $J$, Crostack HA, Rohn K, Witte F, Thorey F, et al. Comparative structural analysis of the canine femoral head in Legg-Calvé-Perthes disease. Vet Radiol Ultrasound. 2009; 50(4):404-411. https://doi.org/10.1111/ j.1740-8261.2009.01557.x

8. Fitzpatrick, N, Pratola, L, Yeadon, R. Total hip replacement after failed femoral head and neck excision in two dogs and two cats. Vet Surg. 2011; 41:136-142. https://doi. org/10.1111/j.1532-950x.2011.00940.x

9. Harper T. Femoral Head and Neck Excision. Veterinary Clinics of North America: Small Animal Practice. 2017; 47:885-897. https:// doi.org/10.1016/j.cvsm.2017.03.002

10. Zhu ZH, Gao YS, Luo SH, Zeng BF, Zhang $C Q$. An animal model of femoral head osteonecrosis induced by a single injection of absolute alcohol: An experimental study. Medical Science Monitor: International Medical Journal of Experimental and Clinical Research. 2011; 17(4):97-102. https://doi. org $/ 10.12659 / \mathrm{msm} .881708$
11. Crawford C, Allen B, Powers D. Growth Profiles and Articular Cartilage Characterization in a Goat Model of Legg-Calvé-Perthes Disease. Journal of Investigative Surgery. 1995; 8:391-408. https://doi. org/10.3109/08941939509031606

12. Tang $T$, Lu $B$, Yue $B$, Xie $X H$, Xie $Y Z$, Dai $\mathrm{KR}$, et al. Treatment of osteonecrosis of the femoral head with hBMP-2-gene-modified tissueengineered bone in goats. The Journal of Bone and Joint Surgery. 2007; 89(1):127132. https://doi.org/10.1302/0301$\underline{620 x .89 b 1.18350}$

13. Harwood D, Mueller K. Goat Medicine and Surgery. CRC Press. 2018. https://www. crcpress.com/Goat-Medicine-and-Surgery/ Harwood-Mueller/p/book/9781498748636\# googlePreviewContainer

14. Kobayashi R, Kurotaki T, Yamada N, Kumabe S, Doi T, Wako Y, Tsuchitani M. Spontaneous and bilateral necrosis of the femoral head in a young experimental beagle dog. Toxicol Pathol. 2015; 28:121-124. https://doi. org/10.1293/tox.2014-0060

15. Winders CLB, Vaughn WL, Birdwhistell $\mathrm{KE}$, Holsworth IG, Franklin SP. Accuracy of femoral head and neck excision via a craniolateral approach or a ventral approach. Veterinary and Comparative Orthopaedics and Traumatology. 2018; 31(2):102-107. https:// doi.org/10.3415/vcot-17-07-0099

16. Off $\mathrm{W}$, Matis $\mathrm{U}$. Excision arthroplasty of the hip joint in dogs and cats. Clinical, radiographic, and gait analysis findings from the Department of Surgery, Veterinary Faculty of the Ludwig-Maximilians-University of Munich, Germany. Vet Comp Orthop Traumatol. 2010; 23(5):297-305. https:// www.orthovet.org/files/vcot 2010-23$\underline{5 \quad 13649 \quad 0 . p d f}$ 